\title{
Extended surgery of antero-superior mediastinum
}

\author{
Vittorio Aprile, Stylianos Korasidis, Diana Bacchin, Marcello Carlo Ambrogi, Marco Lucchi \\ Division of Thoracic Surgery, Cardiac-Thoracic and Vascular Department, University Hospital of Pisa, Azienda Ospedaliero-Universitaria Pisana, \\ Pisa, Italy \\ Contributions: (I) Conception and design: V Aprile, M Lucchi; (II) Administrative support: M Lucchi; (III) Provision of study materials or patients: \\ V Aprile, S Korasidis, MC Ambrogi; (IV) Collection and assembly of data: V Aprile; (V) Data analysis and interpretation: V Aprile; (VI) Manuscript \\ writing: All authors; (VII) Final approval of manuscript: All authors. \\ Correspondence to: Vittorio Aprile. Division of Thoracic Surgery, Cardiac-Thoracic and Vascular Department, University Hospital of Pisa, Azienda \\ Ospedaliero-Universitaria Pisana, via Paradisa 2, 56124 Pisa, Italy. Email: aprilevittorio@gmail.com.
}

\begin{abstract}
Surgery of large mediastinal tumors is a great challenge for the surgeons due to wide number of organs that may be involved despite the narrow space. Any treatment plan should be thoroughly planned step-by-step since the diagnosis to assess the features of the tumor and its relationship with the surrounding organs. Radicality is main goal of the surgeons even if this includes the sacrifice of some structure and the reconstruction of others. Cooperation with the anesthesiologist and surgeons of other speciality is often necessary especially in case of infiltrating tumors. Evolution of the surgical technique end of the peri-operative management as well as the availability of new-generation prosthesis and the increasingly widespread of extracorporeal circulation (ECC) have turned this kind of surgery into a feasible, safe and effective procedure. The cornerstones of this surgery are a detailed preoperative assessment, the ability to prevent any potential complication and involve a multidisciplinary equipe.
\end{abstract}

Keywords: Mediastinal tumors; extended surgery; multidisciplinary board

Received: 07 November 2019; Accepted: 21 November 2019; Published: 19 December 2019.

doi: $10.21037 /$ ccts.2019.11.10

View this article at: http://dx.doi.org/10.21037/ccts.2019.11.10

\section{Introduction}

The mediastinum is a complex region inside the thorax that contains a wide variety of organs and, consequently, may be affected by a great number of disorders. Mediastinum is classically divided into specific compartments. This arbitrary division aids in the definition of differential diagnoses at the initial presentation and facilitates the treatment plans (1). The classical anatomical classification of the mediastinum reports four compartments: anterior, superior, middle and posterior. However, the anterior and the superior compartments are often described as a single anatomical region due to numerous disorders that may affect both concomitantly. The anterior-superior mediastinum is a site for a wide variety of tumors, which arise from its main cellular components, deriving from the thymus, lymph nodes and, rarely, ectopic tissues and embryonic remnants (2). As reported by Takeda et al., the most common location of neoplasia in the mediastinum is the antero-superior compartment that accounts for $68 \%$ of the mediastinal tumors in adults, whereas the posterior mediastinum is more frequently affected in children (3). Tumors of pediatric and adult age show different demographical, clinical and histological spectrum. In infants and children, the primary mediastinal masses in order of decreasing frequency are neurogenic tumors, germ cell tumors (GCTs) and pleuropericardial or enterogenous cysts (4). The most common primary tumor of the antero-superior mediastinum, in adults, are thymomas that accounts for more than $50 \%$ of the mediastinal masses followed by lymphoma, GCT and parathyroid adenoma that rarely required an extended surgery. Nevertheless, the antero-superior mediastinum is, often, involved large metastatic lymph nodes especially in advanced lung and thyroid cancer. Since mediastinal tumors, especially bulky masses, are very uncommon, accounting for less than $5 \%$ of all the tumors, scarce literature is available 
Table 1 Signs and symptoms of mediastinal tumors

\begin{tabular}{ll}
\hline Organ involved & Signs and symptoms \\
\hline Airway & $\begin{array}{l}\text { Cough, wheezing; recurrent respiratory infections, bronchitis, atelectasis, unresolved pneumonia; } \\
\text { hemoptysis; chest pain, sudden death }\end{array}$ \\
Esophagus & Dysphagia (interruption of peristalsis); hematemesis (fistula formation) \\
Superior vena cava & $\begin{array}{l}\text { Dilation of collateral veins of the neck and upper thorax; chemosis of conjunctiva, edema of face, neck, } \\
\text { upper chest, and arm; cyanosis; headaches, visual disturbances; epistaxis, tinnitus }\end{array}$ \\
Lymphatic channels & Pleural effusion \\
Thoracic duct & Chylothorax \\
Recurrent laryngeal nerve & Hoarseness, inspiratory stridor (paralysis of vocal cord) \\
Phrenic nerve & Paralysis of hemi-diaphragm \\
Sympathetic ganglia & Bernard-Horner syndrome \\
Pericardium & Pericardial effusion, tamponade \\
Brachial plexus & Weakness or numbness; loss of sensation; loss of movement (paralysis); pain \\
Vagus nerve & Dysphonia, dysphagia, arrythmia, abnormal blood pressure, gastric stasis (nausea and vomiting), diarrhea \\
\end{tabular}

regarding this matter. Antero-superior large mediastinal tumors offer both diagnostic and therapeutic challenges, due to the complex anatomy of the mediastinum, especially in case of compression or infiltration of vital surrounding structures (5). Since mediastinal space is narrow, up to $60 \%$ of all tumors involving the anterior mediastinum are symptomatic at presentation as consequence of the mechanical compression or infiltration of the neighboring organs (6) as reported in Table 1. In addition, the management of some patients might be further complicated by the mediastinal mass syndrome (MMS) that represents a life-threating condition characterized by acute respiratory and hemodynamic failure, due to obstruction of airways and the compression of the heart or major vessels. This syndrome is a life-threating condition that may became even more dramatic by induction of anesthesia or positional changes during surgery (7). Therefore, a thorough preoperative study is essential to assess the anatomy and exact relationship of the tumor with the surrounding mediastinal structures and to plan, in detail, the eventual operation. Not to forget clinics and/or exams to exclude potential paraneoplastic syndromes associated to the mediastinal masses.

\section{Pre-operative assessment}

The anatomical assessment of the mediastinum is conducted in most cases by the computed tomography (CT) scan of the chest. This examination allows a detailed definition of the tumor's features, from its location to relations with the adjacent structures; moreover, the use of the intravenous contrast highlights the vascularization (8). When needed, further examinations are required to define the relation of the tumors with other structures. The magnetic resonance imaging (MRI) is more useful in soft tissue differentiation and in estimation of the chest wall invasion, while transthoracic echocardiography (TTE), transesophageal echocardiography (TEE) and the more recent electrocardiography (ECG)-gated cardiac CT and MRI improve the assessment of the compression or the involvement of the heart or the main vessels by reducing the cardiac motion artifacts $(9,10)$. Fluorodeoxyglucose positron emission tomography (FDG-PET) has a fundamental role in the diagnostic evaluation of mediastinal tumors, with impact on diagnosis, staging and prognosis but plays a marginal role regarding the anatomical assessment of the mediastinum (11); while tracheo-bronchial tree could be thoroughly evaluated by the bronchoscopy that could be performed also during surgery (12). Large mediastinal tumors are often highly vascularized with a significant risk of bleeding or technical complication during surgery. The blood supply is extremely variable, possibly derived from branches of the internal mammary artery, bronchial arteries, thyrocervical trunk, intercostal vessels, phrenic 
nerve vessels, pulmonary artery, and even from coronary arteries (13-15). MRI or CT scan with intravenous contrast may provide detailed information about the tumor's vascularization and, in case of invasion of the superior vena cava (SVC), on the collateral circulation (16). Recently, some authors advocated the imaging-guided embolization of the tumor's vessels as a useful pre-operative treatment in order to reduce the operative blood loss and, sometimes, the tumor mass, although no large studies are available on this matter $(17,18)$. Preoperative biopsy should be avoided when the anterior mediastinal tumor has well-demarcated borders and could easily be resected completely because the increased possibility of tumor seeding. On the other hand, this approach cannot be used in extended large masses presenting as inoperable cases; where neoadjuvant therapy may improve the resectability or facilitate the surgical resection by "setting free" the surrounding structures involved. Both thymomas and primary mediastinal nonseminomatous GCTs are chemo- and radio-sensitive. When presented as extended mediastinal masses, multimodality treatment may be the approach of choice, consisting of neoadjuvant chemo- or radiation therapy followed by surgical resection of residual disease, furthermore, most of lymphoproliferative malignancies are completely treated by systemic chemotherapy. The role and the effectiveness of preoperative treatment is strictly related to the histopathological diagnosis obtained through a fineneedle aspiration (FNA) and core needle biopsy, or by more invasive surgical procedures for biopsy, including anterior mediastinotomy, mediastinoscopy, and videothoracoscopy. Additional tests such as laboratory studies, the most common of which include $\alpha$-fetoprotein $(\alpha$ $\mathrm{FP}), \beta$-human chorionic gonadotropin ( $\beta$-HCG), and lactate dehydrogenase $(\mathrm{LDH})$ for the non-seminomatous GCTs and antibodies anti-acetylcholine receptor and antimuscle-specific tyrosine kinase for the thymoma related to syndrome myasthenia gravis can play an important role. An excellent work-up scheme has been described by $\mathrm{Li}$ et $\mathrm{al}$. in 2016 (5) and reported with some changes in Figure 1.

\section{Technical aspects}

In case of large mediastinal masses, a good exposure is mandatory to achieve a total control of all structures that may be involved and avoid major complication. Sternotomy is the optimal approach to anterior mediastinal tumors, even in the presence of pulmonary involvement. Any operation on both lung parenchyma, on the main airway or all the great vessels, pericardium could be performed through this approach. Moreover, sternotomy is essential whenever extracorporeal circulation (ECC) is required. This midline approach can be extended to a thoracotomy (hemi-clamshell incision) or may be combined with a cervicotomy where necessary (Figure 2). Rarely have been described other approaches as the thoracotomy or the clamshell incision, while a minimally invasive technique should be considered only with a diagnostic purpose rather than for a radical surgery especially for tumors larger than $10 \mathrm{~cm}(5,19)$. The main goal of extended surgery of anterosuperior mediastinum is be as radical as possible. Absolute contraindications to resection of mediastinal masses are invasion of the myocardium, while the involvement of great vessels or long tracheal segment should be evaluated case-by-case. Any infiltrated structure should be resected and reconstructed when possible. Lung parenchyma could be resected easily, ranging from a wedge resection using a stapler to a lobectomy until a pneumonectomy (eventually, requiring an intrapericardially suture when the hilum is invaded), along with the mediastinal pleura and pericardium. In large pericardial resections a prosthetic mesh should always be placed to avoid the potential migration of the heart through the defect into the thoracic cavities, especially after a pneumonectomy. Phrenic nerve is usually sacrificed when infiltrated, even if it must always be preserved on at least one side, especially if pulmonary resection is planned, but some authors reported an effective technique in sparing the nerve by a gentle peeling of the perineural layers to avoid further respiratory complication, especially in patient with an impaired pulmonary function or myasthenia gravis (20). Of the great vessels, the SVC and the brachiocephalic veins are the most commonly affected, although with varying degrees of involvement, ranging from partial compression or invasion, to the development of SVC syndrome (SVCS) when the vein is completely occluded by the tumor. In general, most authors advocated a total SVC replacement with prosthetic grafts or vein grafts, when the vein is extensively invaded or occluded by the tumor for more than $50 \%$ or when a suture is unfeasible (21). Most authors suggest avoiding SVC reconstruction in the presence of extensive thrombosis invading the subclavian veins due to high risk of thromboembolism or in presence of diffuse collateral venous circulation. If only one brachiocephalic vein is invaded by tumor, it can safely be resected even without replacement (22). Extended operations including resection and reconstruction of the SVC represent a major technical challenge, especially 

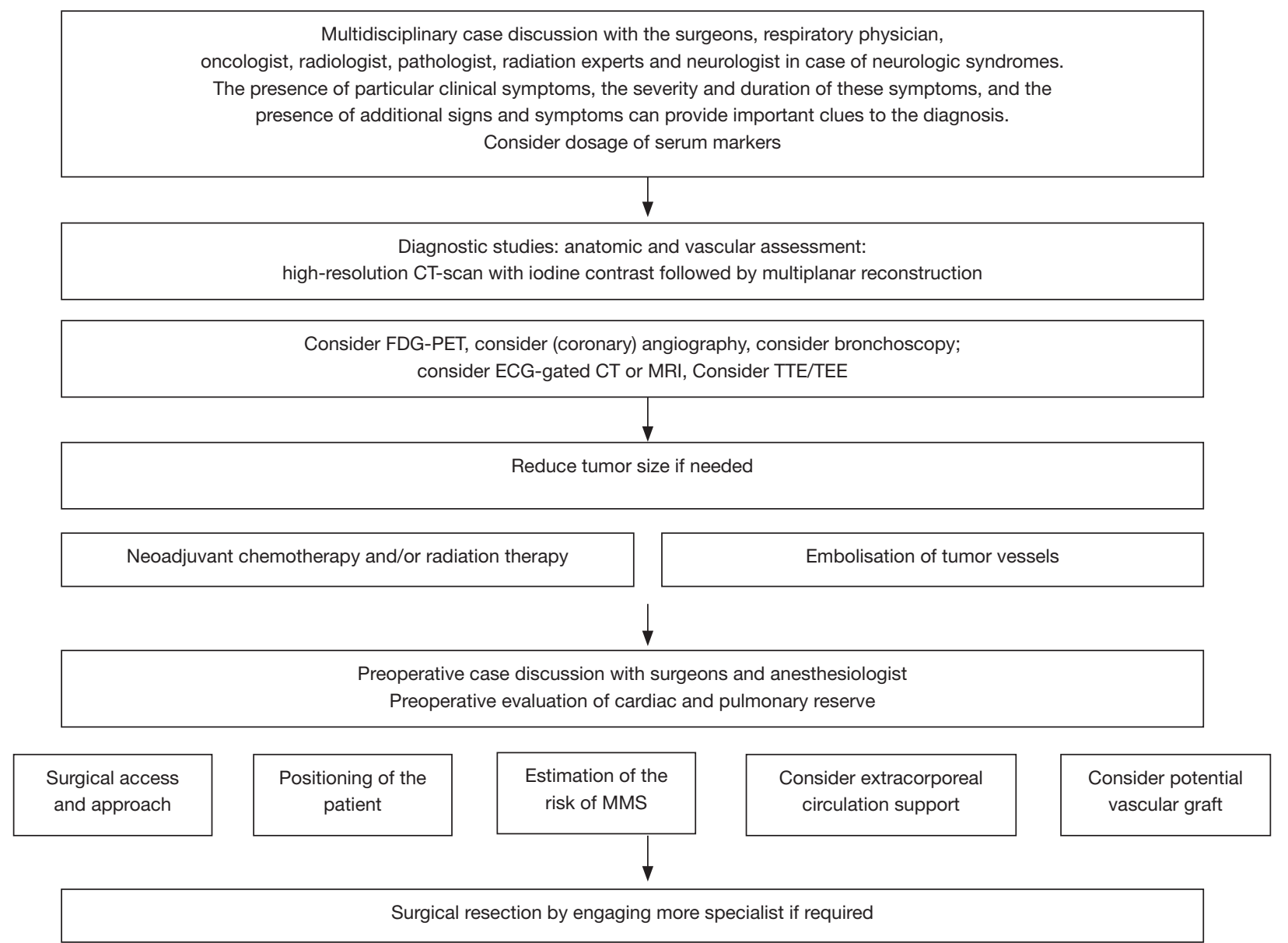

Figure 1 Preoperative assessments of large mediastinal tumors. FDG-PET, fluorodeoxyglucose positron emission tomography; ECG, electrocardiography; CT, computed tomography; MRI, magnetic resonance imaging; TTE, transthoracic echocardiography; TEE, transesophageal echocardiography; MMS, mediastinal mass syndrome.

for the potential detrimental effect of clamping a patent vessel, hence it is essential a close cooperation with the anesthesiologist in charge to manage the patient's hemodynamic as keeping a high cerebral perfusion pressure and administering heparin to prevent potential severe complications. Recently, some authors reported the use of ECC or extra corporeal membrane oxygenation (ECMO) in the surgical management of mediastinal masses $(23,24)$. The assistance of these devices allows an adequate hemodynamic and respiratory function even with lungs deflated and facilitate the dissection of the tumor with a decreased risk of respiratory and hemodynamic decompensation. However, central cannulation may reduce the operative field, hence, peripheral cannulation of the femoral vessels or ECMO should be preferred. However, systemic heparinization required by these devices could increase the risk of hemorrhagic complications during dissection of the tumor as well as the risk of malignant cells spread; therefore, a multidisciplinary risk assessment should be made case-bycase, preoperatively, to assess the need of ECC.

\section{Advanced stage thymoma}

Thymomas occurring as large mediastinal masses are often Masaoka-Koga stage III tumors and represent up to $30 \%$ of all surgically treated thymomas. In most cases occur involving different intrathoracic structures as pericardium, lung parenchyma, great vessels, phrenic nerve, diaphragm and chest wall. Its treatment is still far from being standardized especially due to its rarity and high variability in clinicopathologic behavior (25). In most series the completeness of resection has been reported 


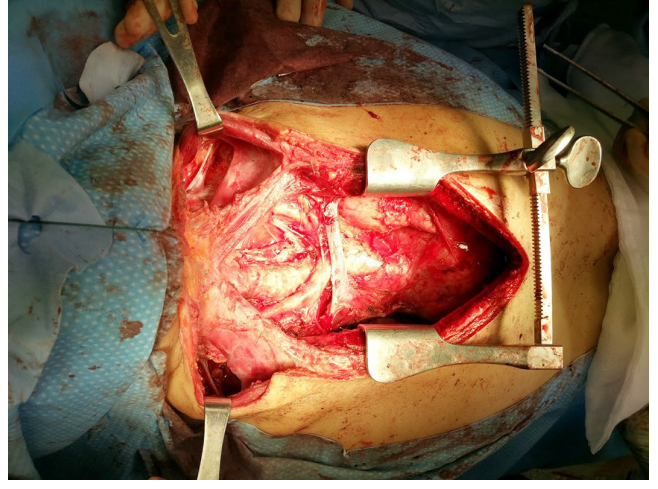

Figure 2 Intraoperative picture of sternotomy extended to cervicotomy to ensure a good exposition during surgery for large tumors of the antero-superior mediastinum invading the cervical compartment.

as the major prognostic factor associated with prolonged local control and survival, but with a reported rate of radical resection highly variable among them (from $50 \%$ to $80 \%)(26,27)$. As reported by Attaran and colleagues, a macroscopic incomplete resection or debulking procedures is associated to an increased recurrence rate and to a poorer prognosis without any advantage compared to biopsy alone (26). In literature several reports of extended surgery and reconstruction for thymic malignancies are reported with excellent results in terms of survival and disease local control. The brachiocephalic veins, SVC, lung, pericardium, right atrium, and diaphragm can be safely resected with or without reconstruction increasing the complete resection rate, in the same way, a resection and reconstruction of the aorta and the main pulmonary artery may be indicated in selected cases to achieve complete resection (1). Recently, a survey conducted by the members of the European Association for Cardio-Thoracic Surgery revealed that neoadjuvant therapy (chemo and/or radiation therapy) is currently proposed in the treatment of thymoma to achieve a volume reduction preoperatively with a complete resection rate, after induction therapy, that reaches the $92 \%$ while 5 -year overall survival (OS) achieves $80 \%$ (27). Beyond the discussion on the effectiveness of the induction therapy, an extended total thymectomy, including the tumor together with resection of any invaded adjacent structure, should be considered the main goal of the surgeons, particularly in the setting of stage III thymomas involving great vessels that occurs from $8.9 \%$ to $24.8 \%$ in major series $(21,25)$. Nevertheless, the involvement of great vessels (brachiocephalic veins, SVC and, even rarely, the aorta and epiaortic vessels) is associated with worse OS (ranging from $45 \%$ to $56 \%$ at 5 years) compared to those patients with invasion of other surrounding structures in case of radical resection (27). The cause of this high difference is probably related to higher risk of intraoperative severe complications and systemic recurrences, as well as the limited chance of a redo-resection (21). Regarding the involvement of phrenic nerve by thymomas some authors advocate a nerve sparing surgery followed by radiation therapy as a valid option especially in myasthenic patients in order to avoid respiratory complications (20).

\section{Mediastinal lymphoma}

Malignant lymphoma may involve the antero-superior mediastinum as a part of disseminated disease or as a primary lesion. The most common types of primary mediastinal lymphomas are Hodgkin's disease (usually of the nodular sclerosis type), large cell lymphoma (often associated with sclerosis), pulmonary mucosa-associated lymphoid tissue lymphoma and lymphoblastic lymphoma (frequently seen in conjunction with acute lymphoblastic leukemia) (28). Clinical presentation may be similar among different subtypes, as most of these neoplasms generally appear as large invasive anterior mediastinal masses with possible involvement of the surrounding structures. Intensive combination of different chemotherapeutic agents and radiation therapy is the primary treatment modality for mediastinal lymphomas, and surgery is mostly confined to diagnostic biopsies when less invasive procedures like FNA or core biopsy are inconclusive. Some authors, moreover, advocated an important role of surgery also in resection of residual masses after systemic therapy. As reported by Bacha and colleagues, resection of such masses has particularly three purposes: it sets out the need of further treatments, eliminates any residual of tumor cells and reduce the need of adjuvant radiotherapy when the tumor mass is completely removed (19).

\section{GCTs}

GCTs of the mediastinum are uncommon neoplasms and contributing to almost $5 \%$ of all mediastinal tumors. These tumors histologically contain the same components as their gonadal counterparts since they are a result of a malignant transformation of arrested germ cells along the urogenital ridge during embryogenesis, but may present different biologic behaviors, clinical characteristics, and poor overall prognoses. They can be divided into three histologic 
categories: mature teratoma, seminomatous GCT, and nonseminomatous GCT (29). Mature teratomas account for about $60 \%$ of all mediastinal GCTs and are characterized by mature tissue derived from all three germinal layers as bone, cartilage, fat, and squamous and glandular epithelia and these tissues may be easily detectable on the CT scan. This kind of tumors are not associated with high level of serum markers like $\alpha$-FP and $\beta$-HCG. Teratoma behavior is extremely variable: the majority present an indolent behavior with adhesion to surrounding structures as main risk, but some mature teratomas can grow more rapidly and may degenerate into non-germ-cell tumors, such as sarcoma and epithelial cancer (30). Surgery is the primary treatment with excellent oncological outcome after complete resection since mature teratomas are not responsive to chemotherapy or radiation therapy. Surgical removal is sometimes challenging especially in case of masses larger than $5 \mathrm{~cm}$ or involving the neighboring organs. It has been estimated that approximately $15 \%$ of patients requires a resection extended to other structures (e.g., lobectomy, pericardiectomy) for complete tumor resection.

\section{Seminomatous GCTs}

The primary mediastinal seminoma is a rare tumor and typically occurs in men from the second to the fourth decade of life. Typically, these lesions present as a lobulated but homogeneous mass and tend to infiltrate into adjacent structures early in the growth process. Serum tumor markers are usually normal even if high levels of $\beta$-HCG can be found in about $40 \%$ of patients. Generally, more than one third of patients with mediastinal seminoma have localized disease at the time of diagnosis, while remaining patients have at least one site of distant metastases. Pure seminoma is sensitive to chemotherapy and radiotherapy, and the prognosis is generally good (31). No conclusive data regarding a potential benefit of primary surgery are available and this approach is usually not feasible at presentation, mainly because the disease is rarely diagnosed as a small mediastinal mass with non-extramediastinal invasion. Pure mediastinal seminomas are curable in most patients, even when metastatic at the time of diagnosis. The treatment of choice is a combination of chemo and radiation therapy (32). It has been shown that a residual mass after chemotherapy frequently does not contain viable tumor cells, hence surgery is recommended only for residual masses larger than $3 \mathrm{~cm}$ with a growing tendency during surveillance.

\section{Non-seminomatous GCTs}

Non-seminomatous GCTs are more common than seminoma and include three histologic subtypes, alone or in combination: yolk sac tumor, embryonal carcinoma, and choriocarcinoma (30). These neoplasms present an aggressive behavior causing, in a short time, symptoms by compressing or invading local mediastinal structures and in more than $85 \%$ at least one metastatic lesion present at the time of diagnosis. Infiltration into adjacent structures including the lung; the SVC; innominate veins; the pericardium; and, occasionally, other great vessels and the heart can be visible on CT scan. Serum tumor markers ( $\alpha$-FP and $\beta-\mathrm{HCG}$ ) are usually abnormal and their high-level in association with typical features on CT scan, is diagnostic of primary malignant non-seminomatous GCTs even without biopsy (32). Approximately 5-10\% of patients affected by mediastinal non-seminomatous GCT is associated to Klinefelter syndrome, more rarely these patients present a variety of hematologic neoplasms. Prognosis is poorer when compared with the other GCTs with an average 5-year OS of almost $50 \%$. The role of surgery is very limited because curative results with other treatment modalities are superior moreover in these tumors is extremely important a multimodal strategy. Most patients have residual radiographic abnormalities in the mediastinum after completion of chemotherapy. In these patients, a radical surgical resection of residual masses should be performed if technically feasible and may be curative in one third of patients (33).

\section{Metastatic lymphnodes}

Metastasis in mediastinal lymph nodes are frequently observed in advanced stage lung cancer, thyroid cancer and more seldom in esophageal, pancreatic, colorectal and breast cancer. Stage III non-small cell lung cancer (NSCLC) describes the metastatic spread of a primary tumor to the ipsilateral and/or contralateral mediastinal lymph nodes and include a wide variety of disease ranging from a single station unsuspected N2 disease to large, bulky and invasive N2 or N3 disease that cannot be dissected (34). Bulky nodal disease is generally defined as involvement of lymph nodes with greater than $2 \mathrm{~cm}$ short-axis diameter as measured by chest CT, and generally implies extra nodal involvement, multi-station nodal disease, and/or groupings of multiple positive smaller lymph nodes (35). For unresectable N2 or N3 NSCLC concurrent chemoradiotherapy is widely accepted as the standard of care while the role of 
surgery has been described in very few reports as part of multimodal treatment, but its effectiveness is still far from being recognized $(36,37)$. Thyroid carcinoma consists essentially of 3 histological types: papillary, follicular and medullary carcinomas. The patterns of metastasis of these 3 carcinomas are different. Follicular carcinoma metastasizes predominantly to distant organs such as lung and bone, whereas papillary and medullary carcinomas shows a high incidence of lymph node metastasis, ranging from $30 \%$ to $80 \%$ of patients, even though metastases to lymph nodes of the upper mediastinum have been found in $9 \%$ of patients with thyroid carcinoma. Thyroid cancer commonly develops regional lymphatic metastases into three regions: central, lateral, and mediastinal compartments. There are lots of researches focused on the central and lateral lymph node metastases so far. However, the indications and extent of the mediastinal lymph node dissection in thyroid carcinoma are not clearly defined (38). In these cases, an accurate multidisciplinary assessment is essential and, in case of failure of systemic therapy, most authors advocate that metastatic mediastinal lymph node dissection is an effective treatment in thyroid carcinoma regardless for initial treatment or reoperation of recurrent lesions (39). Nevertheless, medullary thyroid carcinoma cells are not sensitive to radioactive iodine and to hormonal therapy, and, therefore, surgery is the most effective option either with curative intent or effective palliation. Moreover, in case of metastases, redo-surgery should be considered for patients with elevated calcitonin levels in the setting of an incomplete initial surgery, or if there a radiological evidence of recurrent disease. Iterative surgery should be performed in order to reduce the risk of severe compression or invasion of the airways or major vessels. In experienced hands, reoperation for locoregional disease can achieve long-term control and improve the OS in up to one-third of patients (40).

\section{Conclusions}

Extended surgery of antero-superior mediastinal masses is a real challenge for the surgeons and the anesthesiologists and should be thoroughly planned step-by-step since the diagnosis. The main goal is obtaining a complete resection of the tumor, that remains the major prognostic factor and allows satisfactory long-term survival. Evolution of the surgical technique end of the peri-operative management as well as the availability of new-generation prosthesis and the increasingly widespread of ECC and
ECMO have turned this kind of surgery into a feasible, safe and effective procedure. Centralization of care for patients with these malignancies allows to improve the quality of care that means better surgical and oncological outcome. The preoperative assessment and intraoperative management of these tumors is challenging and a dedicated multidisciplinary approach is essential.

\section{Acknowledgments}

Funding: None.

\section{Footnote}

Provenance and Peer Review: This article was commissioned by the Guest Editors (Francesco Zaraca, Reinhold Perkmann, Luca Bertolaccini and Roberto Crisci) for the Focused Issue "Thoracic Surgery Without Borders" published in Current Challenges in Thoracic Surgery. The article was sent for external peer review organized by the Guest Editors and the editorial office.

Conflicts of Interest: All authors have completed the ICMJE uniform disclosure form (available at http:// dx.doi.org/10.21037/ccts.2019.11.10). The Focused Issue "Thoracic Surgery Without Borders" was commissioned by the editorial office without any funding or sponsorship. The authors have no other conflicts of interest to declare.

Ethical Statement: The authors are accountable for all aspects of the work in ensuring that questions related to the accuracy or integrity of any part of the work are appropriately investigated and resolved.

Open Access Statement: This is an Open Access article distributed in accordance with the Creative Commons Attribution-NonCommercial-NoDerivs 4.0 International License (CC BY-NC-ND 4.0), which permits the noncommercial replication and distribution of the article with the strict proviso that no changes or edits are made and the original work is properly cited (including links to both the formal publication through the relevant DOI and the license). See: https://creativecommons.org/licenses/by-nc-nd/4.0/.

\section{References}

1. D'andrilli A, Rendina EA, Venuta F. Anterior mediastinal lesions. In: Kaiser LR, Jamieson G, Thompson SK. 
editors. Operative thoracic surgery. 6th ed. Boca Raton: CRC Press, 2017.

2. Marino M, Ascani S. An overview on the differential diagnostics of tumors of the anterior-superior mediastinum: the pathologist's perspective. Mediastinum 2019;3:6.

3. Takeda S, Miyoshi S, Akashi A, et al. Clinical spectrum of primary mediastinal tumors: a comparison of adult and pediatric populations at a single Japanese institution. J Surg Oncol 2003;83:24-30.

4. Liu T, Al-Kzayer LFY, Xie X, et al. Mediastinal lesions across the age spectrum: a clinicopathological comparison between pediatric and adult patients. Oncotarget 2017;8:59845-53.

5. Li WW, van Boven WJ, Annema JT, et al. Management of large mediastinal masses: surgical and anesthesiological considerations. J Thorac Dis 2016;8:E175-84.

6. Mullen B, Richardson JD. Primary anterior mediastinal tumors in children and adults. Ann Thorac Surg 1986;42:338-45.

7. Davis RD Jr, Oldham HN Jr, Sabiston DC Jr. Primary cysts and neoplasms of the mediastinum: recent changes in clinical presentation, methods of diagnosis, management, and results. Ann Thorac Surg 1987;44:229-37.

8. Tomiyama N, Honda O, Tsubamoto M, et al. Anterior mediastinal tumors: diagnostic accuracy of CT and MRI. Eur J Radiol 2009;69:280-8.

9. Biederer J, Mirsadraee S, Beer M, et al. MRI of the lung (3/3)-current applications and future perspectives. Insights Imaging 2012;3:373-86.

10. D'Cruz IA, Feghali N, Gross CM. Echocardiographic manifestations of mediastinal masses compressing or encroaching on the heart. Echocardiography 1994;11:523-33.

11. Rankin S. [(18)F]2-fluoro-2-deoxy-D-glucose PET/CT in mediastinal masses. Cancer Imaging 2010;10 Spec no A:S156-60.

12. Kuperberg SJ, Morris BN, Bellinger CR. Anesthetic safety during adult bronchoscopic evaluation of mediastinal masses. Clinical Pulmonary Medicine 2017;24:220-3.

13. Andreini D, Pontone G, Dainese L, et al. Preoperative assessment of thymoma: evaluation of mediastinal arterial anatomy by cardiac multidetector computed tomography. J Thorac Imaging 2009;24:31-3.

14. Morandi U, Stefani A, De Santis M, et al. Preoperative embolization in surgical treatment of mediastinal hemangiopericytoma. Ann Thorac Surg 2000;69:937-9.

15. Allred JD, Mehta D, Courville KA, et al. Mediastinal mass with blood supply from the coronary arteries. Clin Cardiol 2009;32:E49.

16. Eren S, Karaman A, Okur A. The superior vena cava syndrome caused by malignant disease. Imaging with multi-detector row CT. Eur J Radiol 2006;59:93-103.

17. Rakovich G, Ferraro P, Therasse E, et al. Preoperative embolization in the management of a mediastinal paraganglioma. Ann Thorac Surg 2001;72:601-3.

18. Liu FY, Wang MQ, Fan QS, et al. Interventional embolization of giant thoracic tumors before surgical resection. Acta Radiol 2013;54:61-6.

19. Bacha EA, Chapelier AR, Macchiarini P, et al. Surgery for invasive primary mediastinal tumors. Ann Thorac Surg 1998;66:234-9.

20. Aprile V, Bertoglio P, Korasidis S, et al. Nerve-sparing surgery in advanced stage thymomas. Ann Thorac Surg 2019;107:878-84.

21. Leuzzi G, Pastorino U. Extended surgical resection for stage III thymic tumors. Mediastinum 2018;2:40.

22. Detterbeck FC, Parsons AM. Thymic tumors. Ann Thorac Surg 2004;77:1860-9.

23. Vaporciyan AA, Rice D, Correa AM, et al. Resection of advanced thoracic malignancies requiring cardiopulmonary bypass. Eur J Cardiothorac Surg 2002;22:47-52.

24. Wickiser JE, Thompson M, Leavey PJ, et al. Extracorporeal membrane oxygenation (ECMO) initiation without intubation in two children with mediastinal malignancy. Pediatr Blood Cancer 2007;49:751-4.

25. Marulli G, Lucchi M, Margaritora S, et al. Surgical treatment of stage III thymic tumors: a multi-institutional review from four Italian centers. Eur J Cardiothorac Surg 2011;39:e1-7.

26. Attaran S, Acharya M, Anderson JR, et al. Does surgical debulking for advanced stages of thymoma improve survival? Interact Cardiovasc Thorac Surg 2012;15:494-7.

27. Lucchi M, Van Schil P, Schmid R, et al. Thymectomy for thymoma and myasthenia gravis. A survey of current surgical practice in thymic disease amongst EACTS members. Interact Cardiovasc Thorac Surg 2012;14:765-70.

28. Strickler JG, Kurtin PJ. Mediastinal lymphoma. Semin Diagn Pathol 1991;8:2-13.

29. Takeda S, Miyoshi S, Ohta M, et al. Primary germ cell tumors in the mediastinum: a 50-year experience at a single Japanese institution. Cancer 2003;97:367-76.

30. Moran CA, Suster S. Primary germ cell tumors of the mediastinum: I. Analysis of 322 cases with special emphasis on teratomatous lesions and a proposal for histopathologic 
classification and clinical staging. Cancer 1997;80:681-90.

31. Rosti G, Secondino S, Necchi A, et al. Primary mediastinal germ cell tumors. Seminars in oncology. Philadelphia: WB Saunders, 2019.

32. Hainsworth JD, Greco FA. Primary germ cell tumors of the thorax. In: Kufe DW, Pollock RE, Weichselbaum RR, et al. Holland-Frei cancer medicine. 6th ed. Hamilton: BC Decker, 2003.

33. Saxman SB, Nichols CR, Einhorn LH. Salvage chemotherapy in patients with extragonadal nonseminomatous germ cell tumors: the Indiana University experience. J Clin Oncol 1994;12:1390-3.

34. Evison M, McDonald F, Batchelor T. What is the role of surgery in potentially resectable N2 non-small cell lung cancer? Thorax 2018;73:1105-9.

35. Robinson LA, Ruckdeschel JC, Wagner H Jr, et al. Treatment of non-small cell lung cancer-stage IIIA: ACCP evidence-based clinical practice guidelines (2nd edition). Chest 2007;132:243S-65S.

doi: $10.21037 /$ ccts.2019.11.10

Cite this article as: Aprile V, Korasidis S, Bacchin D, Ambrogi MC, Lucchi M. Extended surgery of antero-superior mediastinum. Curr Chall Thorac Surg 2019;1:21.
36. Sakuraba M, Takahashi N, Oh S, et al. Long-term survival after complete mediastinal lymph node resection and lobectomy in patients with bulky N2 non-small cell lung cancer. Ann Thorac Cardiovasc Surg 2011;17:124-9.

37. Yokomise H, Gotoh M, Okamoto T, et al. Induction chemoradiotherapy (carboplatin-taxane and concurrent 50-Gy radiation) for bulky cN2, N3 non-small cell lung cancer. J Thorac Cardiovasc Surg 2007;133:1179-85.

38. Yamashita H, Masatsugu T, Uchino S, et al. Crank-shaped sternotomy for upper mediastinal lymph node dissection in patients with differentiated thyroid cancer. Surg Today 2004;34:480-1.

39. Zhang TT, Qu N, Hu JQ, et al. Mediastinal lymph node metastases in thyroid cancer: characteristics, predictive factors, and prognosis. Int J Endocrinol 2017;2017:1868165.

40. Jin LX, Moley JF. Surgery for lymph node metastases of medullary thyroid carcinoma: a review. Cancer 2016;122:358-66. 